\title{
Serotonin 2C Antagonism in the Lateral Orbitofrontal Cortex Ameliorates Cue-Enhanced Risk Preference and Restores Sensitivity to Reinforcer Devaluation in Male Rats
}

\author{
Bett A. Hathaway, Jackson D. Schumacher, Kelly M. Hrelja, and Catharine A. Winstanley
}

https://doi.org/10.1523/ENEURO.0341-21.2021

Department of Psychology, Djavad Mowafaghian Centre for Brain Health, University of British Columbia, Vancouver, British Columbia V6T 1Z3, Canada

\begin{abstract}
Previous research has indicated that reward-paired cues can enhance disadvantageous risky choice in both humans and rodents. Systemic administration of a serotonin $2 \mathrm{C}$ receptor antagonist can attenuate this cue-induced risk preference in rats. However, the neurocognitive mechanisms mediating this effect are currently unknown. We therefore assessed whether the serotonin 2C receptor antagonist RS 102221 is able to attenuate cue-enhanced risk preference via its actions in the lateral orbitofrontal cortex (IOFC) or prelimbic (PrL) area of the medial prefrontal cortex (mPFC). A total of 32 male Long-Evans rats were trained on the cued version of the rat gambling task (rGT), a rodent analog of the human lowa gambling task, and bilateral guide cannulae were implanted into the IOFC or PrL. Intra-IOFC infusions of the 5- $\mathrm{HT}_{2 \mathrm{C}}$ antagonist RS 102221 reduced risky choice in animals that showed a preference for the risky options of the rGT at baseline. This effect was not observed in optimal decision-makers, nor those that received infusions targeting the PrL. Given prior data showing that $5-\mathrm{HT}_{2 \mathrm{C}}$ antagonists also improve reversal learning through the same neural locus, we hypothesized that reward-concurrent cues may amplify risky decision-making through cognitive inflexibility. We therefore devalued the sugar pellet rewards used in the cued rGT (crGT) through satiation and observed that decision-making patterns did not shift unless animals also received intra-IOFC RS 102221. Collectively, these data suggest that the IOFC is one critical site through which reward-concurrent cues promote risky choice patterns that are insensitive to reinforcer devaluation, and that $5-\mathrm{HT}_{2 \mathrm{C}}$ antagonism may optimize choice by facilitating exploration.
\end{abstract}

Key words: decision making; flexibility; orbitofrontal cortex; reward; risk; serotonin

\section{Significance Statement}

Lights and sounds signaling reward are used extensively in electronic gaming machines. Recent data indicate that these win-associated cues can increase disadvantageous risky choice. Administering a serotonin $2 \mathrm{C}$ receptor antagonist can ameliorate this effect in rats, potentially by increasing flexibility in decision-making. The orbitofrontal cortex (OFC) is critically involved in mediating flexible behavior. Thus, the present study evaluated whether serotonin $2 \mathrm{C}$ antagonism in the OFC can reduce disadvantageous risky choice via alterations to behavioral flexibility. Results implicate the OFC as one critical locus, and an increase in flexibility as a potential cognitive mechanism, through which cue-enhanced risky decision-making may improve. This could point to potential therapeutic interventions for problematic gambling that target the control of cues over behavior. 


\section{Introduction}

Win-associated audiovisual cues are used extensively in electronic games, smartphone apps, and commercial gambling products. Although these cues seem harmless, this form of sensory enhancement can impair decision-making. Specifically, adding win-concurrent cues to laboratory-based gambling tasks increases risky choice in rats and humans (Barrus and Winstanley, 2016; Cherkasova et al., 2018). Risky decision-making can contribute to the onset and maintenance of addiction disorders (Bolla et al., 2003; Goudriaan et al., 2005; Stevens et al., 2013). As such, the ability of rewardsynchronous cues to increase risky choice may facilitate the development of pathologic gambling. Determining the neural and cognitive basis of this effect may shed light on how electronic games can become addictive and identify potential therapeutic interventions.

In rats, we have studied cue-driven risky choice using the rat gambling task (rGT), loosely analogous to the lowa gambling task used clinically (Bechara et al., 1994; Zeeb et al., 2009). In both tasks, maximal reward is attained by avoiding the high-risk, high-reward options and instead favoring the options associated with lower per-trial gains. On the rGT, these low-risk, low-reward options result in less frequent and shorter time-out penalties and therefore more sugar pellets are earned overall. The addition of reward-paired audiovisual cues leads to greater risky choice on average (Barrus and Winstanley, 2016). Decision-making on the cued rGT (crGT) is subject to unique pharmacological regulation. Systemic administration of a serotonin (5-HT) $2 \mathrm{C}$ receptor antagonist SB242084 decreased risky choice selectively on the crGT while increasing premature responding, a reliable index of motor impulsivity, on both the cued and uncued versions of the task (Adams et al., 2017).

This finding was somewhat unexpected, given the previously described role of the $5-\mathrm{HT}_{2 \mathrm{C}}$ receptor in cue-mediated behaviors such as cue-induced reinstatement of cocaine seeking and responding for a conditioned reinforcer (Pentkowski et al., 2010; Browne et al., 2017). Modulation of both mesolimbic dopamine release and activity within the medial prefrontal cortex (mPFC) were

Received August 19, 2021; accepted November 16, 2021; First published November 23, 2021.

In the past three years, C.A.W. has acted as an expert witness for Hogan Lovells LLP and received due compensation. All other authors declare no competing financial interests.

Author contributions: B.A.H., J.D.S., and C.A.W. designed research; B.A.H., J.D.S., and K.M.H. performed research; B.A.H. and C.A.W. analyzed data; B.A.H. and C.A.W. wrote the paper.

This work was supported by the Canadian Institutes for Health Research (CIHR) Grant PJT-162312 (to C.A.W.). B.A.H. was supported by a Doctoral Award from $\mathrm{ClHR}$

Acknowledgements: University of British Columbia is situated on the traditional, ancestral, and unceded land of the $x^{w} m ə \theta k^{w}$ əy่วm (Musqueam), sảlílwotar1/Selilwitulh (Tsleil-Waututh), and Skxwxwú7mesh (Squamish) Peoples. We thank them for their stewardship of this land for thousands of years.

Correspondence should be addressed to Brett A. Hathaway at bretthathaway@ psych.ubc.ca or Catharine A. Winstanley at cwinstanley@psych.ubc.ca.

https://doi.org/10.1523/ENEURO.0341-21.2021 Copyright @ 2021 Hathaway et al.

This is an open-access article distributed under the terms of the Creative Commons Attribution 4.0 International license, which permits unrestricted use, distribution and reproduction in any medium provided that the original work is properly attributed. implicated in these results, and the findings were attributed to alterations in the incentive salience of the cues. Increased premature responding induced by $5-\mathrm{HT}_{2 \mathrm{C}}$ antagonism on the rGT (Adams et al., 2017) and the 5choice serial reaction time task (5CSRT; Winstanley et al., 2004) may also depend on the nucleus accumbens, as systemic administration enhances accumbal dopaminergic release (Browne et al., 2017), which can increase this form of impulsivity (Pattij et al., 2007; Economidou et al., 2012). Indeed, infusions of a $5-\mathrm{HT}_{2} \mathrm{C}$ antagonist into the accumbens of rats increased premature responding on the 5CSRT, whereas microinjections into the mPFC had little effect (Robinson et al., 2008).

These findings suggest that $5-\mathrm{HT}_{2} \mathrm{C}$ receptor antagonism in the nucleus accumbens would enhance the control of cues over behavior, and $5-\mathrm{HT}_{2 \mathrm{C}}$ agonism would attenuate it. However, we instead found that systemic administration of the antagonist ameliorated the risk-enhancing effect of cues on the rGT, while the agonist was without effect. As such, 5- $\mathrm{HT}_{2 \mathrm{C}}$ antagonism may mitigate cue-driven risky choice through a different mechanism than described above, and distinct neural loci.

5-HT, particularly the $5-\mathrm{HT}_{2 \mathrm{C}}$ receptor, is critically involved in mediating flexible behavior (Barlow et al., 2015). Administration of a 5- $\mathrm{HT}_{2 \mathrm{C}}$ antagonist into rats' lateral orbitofrontal cortex (IOFC) but not the mPFC reduced perseveration during reversal learning (Boulougouris and Robbins, 2010). Several studies have identified the IOFC as a critical region for flexibility in decision-making (Baxter et al., 2000; Schoenbaum et al., 2002; Izquierdo et al., 2004; Amodeo et al., 2017). On the uncued rGT, the IOFC is involved in determining the optimal decision-making strategy (Zeeb and Winstanley, 2011). Interestingly, inactivating the IOFC during acquisition of the crGT may increase optimal choice (Ferland, J.-M. N., Barrus, M. M., Betts, G. D., and Winstanley, C. A., unpublished observations). As such, the inclusion of cues may impair decision-making by altering the establishment of accurate action-outcome contingencies in the IOFC. Theoretically, manipulating serotonergic activity in this region could reintroduce flexibility in the stored actionoutcome contingencies and thereby ameliorate this effect.

We therefore administered the $5-\mathrm{HT}_{2 \mathrm{C}}$ antagonist $\mathrm{RS}$ 102221 directly into the IOFC and assessed performance on the crGT. To confirm the regional specificity of any observed effects, we also targeted the prelimbic (PrL) region of the mPFC. We hypothesized that intra-IOFC, but not intra-PrL, RS 102221 would attenuate risky decision-making. We also tested whether decision-making on the crGT is less flexible compared with the uncued rGT by evaluating sensitivity to reinforcer devaluation. Finally, we investigated whether intra-IOFC RS 102221 could restore sensitivity to this manipulation, as expected if $5-\mathrm{HT}_{2 \mathrm{C}}$ antagonism improves decision-making by reinstating flexibility.

\section{Materials and Methods}

\section{Subjects}

Subjects were 48 male Long-Evans rats (Charles River Laboratories) weighing 275-300 g on arrival to the facility. 
One to two weeks following arrival, rats were food-restricted to $14 \mathrm{~g}$ of rat chow per day and were maintained at least $85 \%$ body weight of an age-matched and sexmatched control (initial weight before food restriction: $M=353 \mathrm{~g}, \mathrm{SD}=51 \mathrm{~g}$; weight before surgery: $M=399 \mathrm{~g}$, $\mathrm{SD}=31 \mathrm{~g}$ ). Water was available ad libitum. All subjects were pair-housed or trio-housed in a climate-controlled colony room under a $12 / 12 \mathrm{~h}$ reverse light/dark cycle $\left(21^{\circ}\right.$ $\mathrm{C}$; lights off at 8 A.M.). Huts and paper towel were provided as environmental enrichment. Behavioral testing took place $5 \mathrm{~d}$ per week. Housing and testing conditions were in accordance with the Canadian Council of Animal Care, and experimental protocols were approved by the UBC Animal Care Committee.

\section{Behavioral apparatus}

Testing took place in 32 standard five-hole operant chambers, each of which was enclosed in a ventilated, sound-attenuating chamber (Med Associates Inc). Chambers were fitted with an array composed of five equidistantly spaced response holes. A stimulus light was located at the back of each hole, and nose-poke responses into these apertures were detected by vertical infrared beams. On the opposite wall, sucrose pellets ( $45 \mathrm{mg}$; Bioserv) were delivered to the magazine via an external pellet dispenser. The food magazine was also fitted with a tray light and infrared sensors to detect sucrose pellet collection. A house light could illuminate the chamber. The operant chambers were operated by software written in Med-PC by CAW, running on an IBM-compatible computer.

\section{crGT training and testing}

Details of training and testing have been reported previously (Zeeb et al., 2009; Barrus and Winstanley, 2016). Rats were first habituated to the operant chambers in two daily 30-min sessions, during which sucrose pellets were present in the nose-poke apertures and food magazine. Rats were then trained on a variant of the 5CSRT (Carli et al., 1983), in which rats were required to make a nosepoke response in one of the four apertures, indicated by a $10 \mathrm{~s}$ stimulus light. A correct response was rewarded by delivery of one sugar pellet to the food magazine. The location of the stimulus light varied between holes 1, 2, 4, and 5 across the session. Sessions lasted $30 \mathrm{~min}$ and consisted of $\sim 100$ trials. Rats were trained until they reached a criteria of $\geq 50$ correct responses with $\geq 80 \%$ accuracy and $\leq 20 \%$ omissions. Rats were then trained on a forced-choice variant of the crGT for seven sessions, in which rats were presented with one of the four options per trial in a pseudo-random fashion. This ensured rats had equal exposure to each reinforcement contingency before training on the free-choice version of the program.

A task schematic of the crGT is provided in Figure 1. During the 30 -min session, trials were initiated by a nosepoke response within the illuminated food magazine. This response extinguished the light and started a 5-s intertrial interval (ITI). Any response at the five-hole array during the ITI was recorded as a premature response and

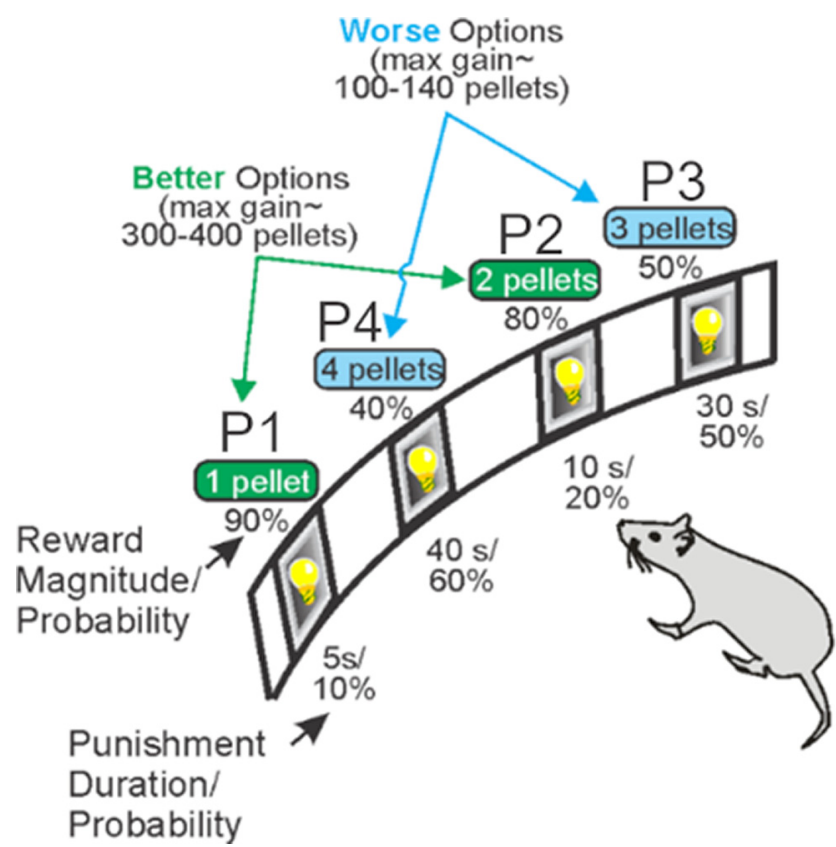

Figure 1. Task schematic of the crGT. A nose poke response in the food tray extinguished the traylight and initiated a new trial. After an ITI of $5 \mathrm{~s}$, four stimulus lights were turned on in holes $1,2,4$, and 5, each of which was associated with a different number of sugar pellets. The order of the options from left to right was counter-balanced within each cohort to avoid development of a simple side bias [version A (shown): P1, P4, P3, $\mathrm{P} 2$; version $\mathrm{B}$ : P4, P1, P3, P2]. The animal was required to respond at a hole within $10 \mathrm{~s}$. This response was then rewarded or punished depending on the reinforcement schedule for that option. Reward delivery was accompanied by a 2-s audiovisual cue that increased in complexity with reward magnitude. If the animal lost, the stimulus light in the chosen hole flashed at a frequency of $0.5 \mathrm{~Hz}$ for the duration of the punishing time out, and all other lights were extinguished. The maximum number of pellets available per 30 -min session shows that $\mathrm{P} 1$ and $\mathrm{P} 2$ are better than P3 and P4. The percent choice of the different options is one of the primary dependent variables. A score variable is also calculated, as for the IGT, to determine the overall level of risky choice as follows: [(P1 + P2) - (P3 + P4)]. Figure is modified from Barrus and Winstanley (2016).

punished by a 5-s time-out period, during which the house light was illuminated and no reward could be earned.

Following the ITI, apertures 1, 2, 4, and 5 in the five-hole array were illuminated for $10 \mathrm{~s}$. If the rat failed to nosepoke in any illuminated hole within $10 \mathrm{~s}$, the trial was recorded as an omission, the food magazine was re-illuminated, and rats were required to initiate a new trial. A nose-poke response within an illuminated aperture was either rewarded or punished according to that aperture's reinforcement schedule. Probability of reward varied among options (0.9-0.4, P1-P4), as did reward size (one to four sucrose pellets). Punishments were signaled by the light within the chosen aperture flashing at a frequency of $0.5 \mathrm{~Hz}$, which lasted for a 5- to 40 -s time-out penalty depending on the aperture selected. Two-second compound tone/light cues occurred concurrently with 
reward delivery. Cue complexity and variability scaled with reward size. The task was designed such that the optimal strategy to earn the highest number of sucrose pellets during the $30-\mathrm{min}$ session would be to exclusively select the P2 option, because of the relatively high probability of reward (0.8) and short, infrequent time-out penalties (10 s, 0.2 probability). While options P3 and P4 provide higher per-trial gains of three or four sucrose pellets, the longer and more frequent time-out penalties associated with these options greatly reduces the occurrence of rewarded trials, such that consistently selecting these options results in fewer sucrose pellets earned across the session and are therefore considered disadvantageous.

The position of each option for the crGT was counterbalanced across rats such that half the animals were trained on version A (left to right arrangement: P1, P4, P2, $P 3$ ) and the other half on version $B$ (left to right arrangement: P4, P1, P3, P2) to mitigate potential side bias. Rats received five training sessions per week.

\section{Surgery}

When baseline performance was deemed statistically stable (following $\sim 40$ training sessions), 32 animals were anesthetized with $2 \%$ isoflurane in $\mathrm{O}_{2}$ and 23-gauge stainless steel guide cannulae were implanted above the IOFC $(n=20 ; \mathrm{AP}=+3.5 \mathrm{~mm}, \mathrm{ML}= \pm 2.6 \mathrm{~mm}$ from breg$\mathrm{ma}, \mathrm{DV}=-2.9 \mathrm{~mm}$ from dura) or the $\operatorname{PrL}(n=12 ; \mathrm{AP}=$ $+3.0 \mathrm{~mm}, \mathrm{ML}= \pm 0.7 \mathrm{~mm}$ from bregma, $\mathrm{DV}=-2.8 \mathrm{~mm}$ from dura), using standard stereotaxic techniques. Guide cannulae were fixed to the skull via four stainless steel screws and dental acrylic, and obdurators flush with the end of the cannulae were inserted. Animals were given at least one week of recovery in their home cages before subsequent testing.

\section{Drug preparation}

Three concentrations of the compound RS 102221 hydrochloride (Tocris Bioscience) were prepared each dosing day. First, $1 \mathrm{mg}$ of the drug was suspended in $300 \mu \mathrm{l}$ of $0.1 \mathrm{M} \mathrm{HCl}$ via sonication. The $\mathrm{pH}$ level was then adjusted to $6-7$ with 1.0 and $0.1 \mathrm{~m} \mathrm{NaOH}$ and saline to a final concentration of $2 \mathrm{mg} / \mathrm{ml}$. Two aliquots of the solution were further diluted to 0.2 and $0.6 \mathrm{mg} / \mathrm{ml}$. The highest concentration dose was vortexed before each infusion to prevent precipitation of the drug during the procedure. The vehicle solution consisted of saline that was $\mathrm{pH}$-adjusted to 6-7 with $\mathrm{NaOH}$.

\section{Microinfusion procedure}

Following recovery, animals performed 10 free-choice sessions, after which all individuals displayed stable behavior. Animals were then habituated to the microinfusion process with two mock infusions, during which 30-gauge dummy injectors were inserted for 2 min but no infusion was performed, followed by a behavioral testing session initiated $10 \mathrm{~min}$ later. Infusions adhered to a $3-d$ cycle starting with a baseline session, followed by a drug or vehicle injection session, and then by a non-testing day; 0.5 $\mu \mathrm{l}$ per hemisphere injections of saline or RS 102221 (0.1,
0.3 , or $1.0 \mu \mathrm{g}$ of drug per hemisphere) were administered bilaterally at a rate of $0.3 \mu \mathrm{l} / \mathrm{min}$ with injectors that extended $0.8 \mathrm{~mm}$ beyond the guide cannulae. Injectors were left in place for an additional minute to allow for diffusion. Animals received each dose of RS 102221 plus vehicle, counterbalanced in a Latin Square design (for doses A thru D: ABCD, CADB, BDAC, DCBA). Once the microinfusions were completed, injectors were removed, obdurators replaced, and animals were placed in the operant chambers for $10 \mathrm{~min}$ before initiation of the crGT.

\section{Reinforcer devaluation}

Twelve IOFC-cannulated rats and 16 surgically-naive rats underwent a reinforcer devaluation procedure. For the naive rats, this procedure took place across $2 \mathrm{~d}$. On the first day, half of the rats were given ad libitum access to the sucrose pellets used as a reward on the crGT for 1 $\mathrm{h}$ before task initiation. The remaining rats completed the crGT without prior access to sucrose pellets. Following a baseline session day for which no sucrose pellets were administered before the task to any rats, the groups were then reversed and the other half were given 1-h access to sucrose pellets. To prevent the accumulation of damage of multiple infusions from impacting the results of this procedure, only one session of reinforcer devaluation was completed for cannulated rats. Ten minutes before task initiation, half of the rats received $1.0 \mu \mathrm{g}$ of RS $102221 \mathrm{per}$ hemisphere, according to the microinfusion procedure specified above. The other half received a vehicle dose. All rats in this group were given ad libitum access to sucrose pellets for $1 \mathrm{~h}$ before task initiation.

\section{Histology}

Following completion of all behavioral testing, animals were anesthetized with isoflurane and euthanized by carbon dioxide exposure. Brains were extracted and fixed in $4 \%$ formaldehyde for at least $24 \mathrm{~h}$, transferred to a $30 \%$ sucrose solution, and then frozen and cut via cryostat into $40-\mu \mathrm{m}$ coronal sections. These sections were stained with cresyl violet for visualization, and the projected locations of the injector tips protruding from the guide cannulae were mapped onto standard sections from Paxinos and Watson (1998).

\section{Behavioral measures and data analysis}

All statistical analyses were completed using SPSS Statistics 27.0 software (SPSS/IBM). As per previous reports, the following rGT variables were analyzed: percentage choice of each option (number of times option chosen/total number of choices $\times 100$ ), risk score (calculated as percent choice of $[(\mathrm{P} 1+\mathrm{P} 2)-(\mathrm{P} 3+\mathrm{P} 4)])$, percentage of premature responses (number of premature responses/total number of trials initiated $\times 100$ ), sum of omitted responses, sum of trials completed, and average latencies to choose an option and collect reward. Variables that were expressed as a percentage were subjected to an arcsine transformation to limit the effect of an artificially imposed ceiling (i.e., 100\%). A statistically stable baseline was determined by a 


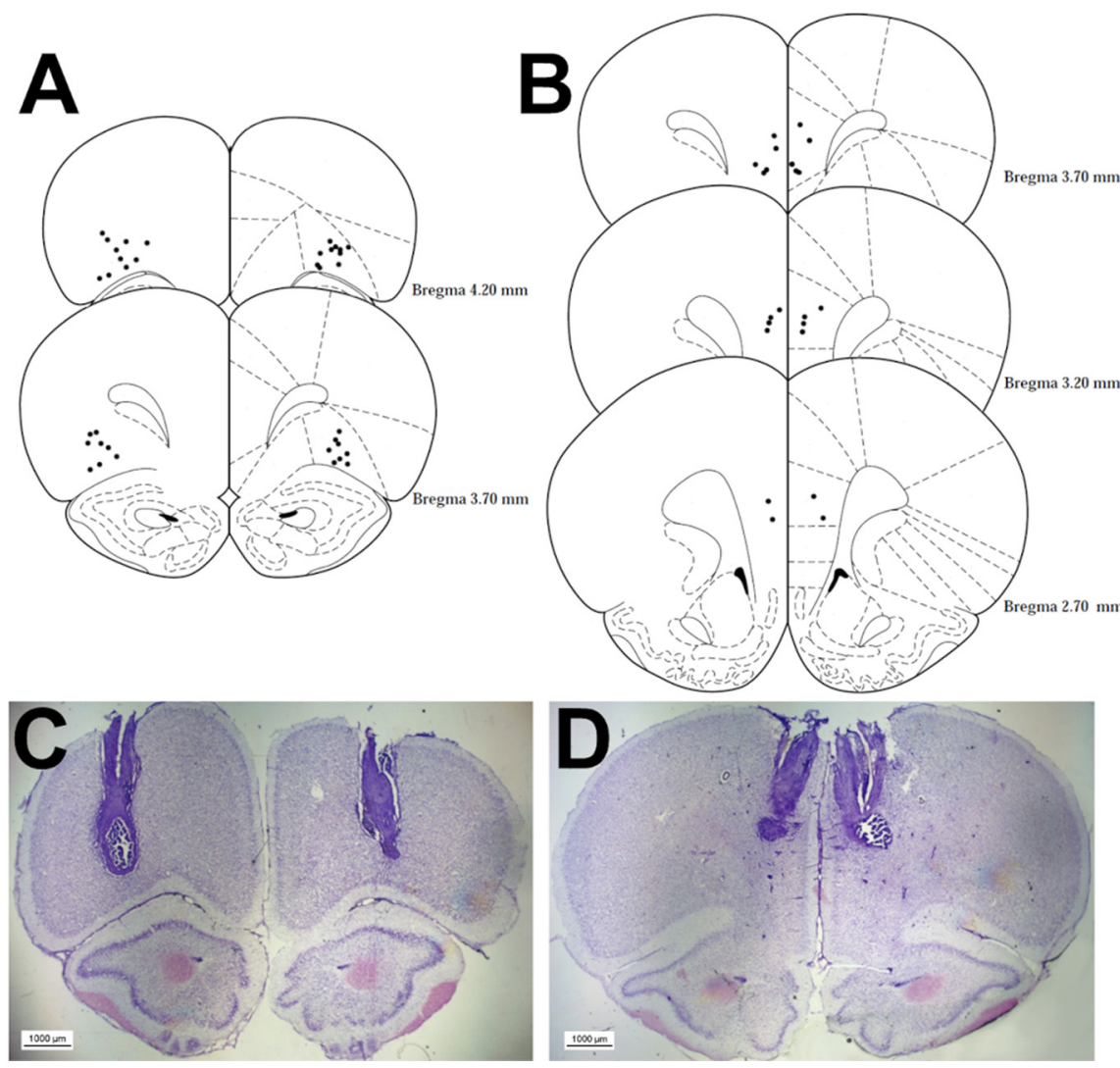

Figure 2. Histologic analysis of cannulae implantation. Location of all acceptable IOFC $(\boldsymbol{A})$ and $\operatorname{PrL}$ region $(\boldsymbol{B})$ infusions and an example microscopy image for each region in $\boldsymbol{C}, \boldsymbol{D}$ are shown. Coordinates are relative to bregma. Plates modified from Paxinos and Watson (1998).

repeated-measures ANOVA across data from four consecutive sessions before surgery, following $\sim 40$ training sessions, in which both the session factor and session $x$ choice interaction were not significant. Animals with a mean positive baseline risk score were designated as "optimal," whereas rats with negative risk scores were classified as "risk-preferring."

Choice data were analyzed with a two-way repeated measures ANOVA with dose (four levels: vehicle, $0.1 \mu \mathrm{g}$, $0.3 \mu \mathrm{g}$, and $1.0 \mu \mathrm{g}$ ) and choice (four levels: P1, P2, P3, and $\mathrm{P} 4$ ) as within-subject factors. For all other variables, dose was the only within-subjects factor. Risk status (two levels: optimal, risk-preferring) was included as a between-subjects factor for all statistical analyses. For the analysis of the reinforcer devaluation data, devaluation (two levels: baseline, devaluation) and choice (four levels: P1-P4) were the within-subject factors and group (three levels: surgically-naive, vehicle, drug) and risk status were the between-subjects factors. The baseline session used for each group was as follows: naive rats, session without experimental manipulation (i.e., no devaluation); vehicle rats, vehicle data from Latin Square dosing regimen; drug rats, highest concentration dose data from Latin Square dosing regimen. In isolated cases where data were missing because of technical issues, mean replacements were used.

For all analyses, if sphericity was violated as determined by Mauchley's test, a Huynh-Feldt correction was applied, and corrected $p$ values' degrees of freedom were rounded to the nearest integer. Results were deemed to be significant if $p$ values were less than or equal to an $\alpha$ of .05. Any main effects or interactions of significance were further analyzed via post hoc one-way ANOVA or paired samples $t$ tests with a Bonferroni correction applied for the number of comparisons made. Any $p>0.05$ but $p<$ 0.09 were reported as a statistical trend.

\section{Results}

\section{Cannulae placements}

The locations of all acceptable placements are depicted in Figure $2 A$ for the IOFC cohort and Figure $2 B$ for the PrL cohort. One animal in the IOFC condition did not survive surgery. One rat was excluded from the IOFC analyses because of inaccurate placement of the cannulae. All PrL cannulae placements were acceptable. This left a total of 18 ( $n=9$ risk-preferring; $n=9$ optimal) and 12 ( $n=6$ riskpreferring; $n=6$ optimal) rats for the IOFC and PrL analyses, respectively.

\section{Baseline behavior}

One rat was excluded from the PrL condition because of poor task performance (mean of 30 trials completed per session, $>30$ s reward collection latency). As expected, risk- 


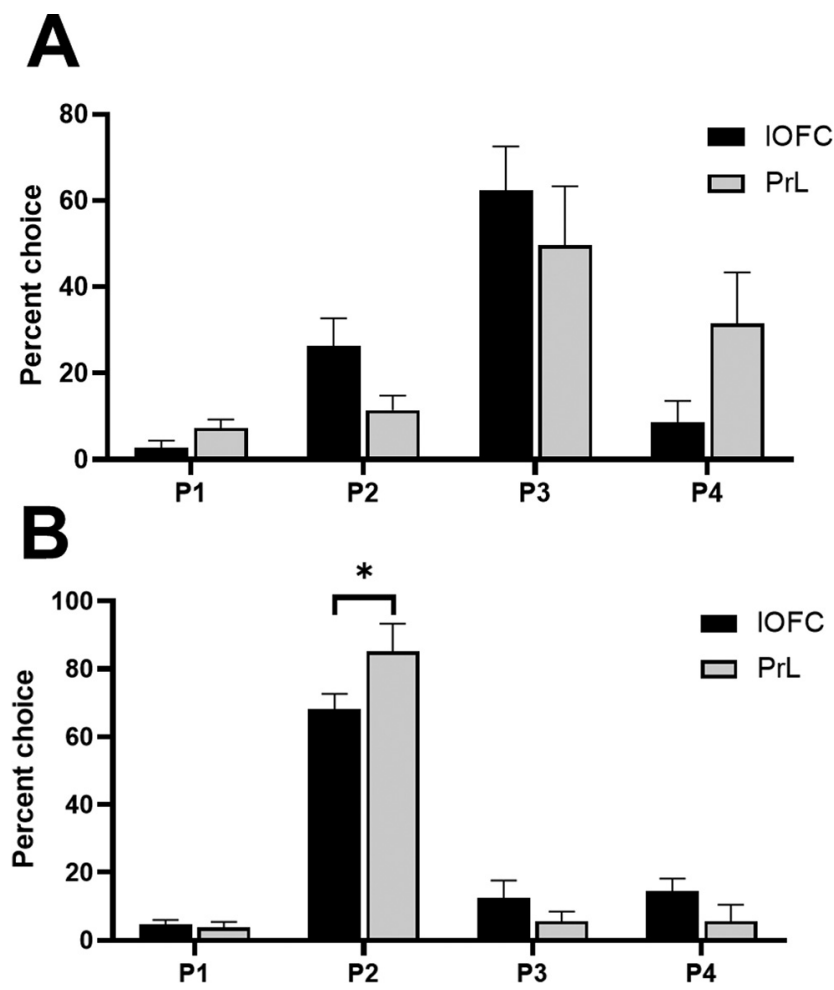

Figure 3. Baseline preferences for each option in the IOFC and PrL cohorts in risk-preferring rats $(\boldsymbol{A})$ and optimal decision-makers $(\boldsymbol{B})$. Optimal rats in the PrL cohort selected P2 at a significantly higher rate than optimal rats in the IOFC cohort. Data are expressed as mean \pm SEM; ${ }^{*} p<0.05$ according to an independent-groups $t$ test.

preferring rats selected the risky options at a significantly higher proportion than those who developed an optimal decision-making strategy for all baseline and saline sessions (choice $\times$ risk preference: $F_{(2,148)}=41.77, p<0.0001$; optimal vs risk-preferring: $\mathrm{P} 1: t_{(75)}=1.60, p=0.11 ; \mathrm{P} 2: t_{(75)}=12.89$, $p<0.0001$; P3: $t_{(68)}=-5.95, p<0.0001 ; \mathrm{P} 4: t_{(66)}=-3.97$, $p=0.0002)$. Across both cohorts, risk-preferring rats completed significantly fewer trials (risk preference: $F_{(1,25)}=38.43$, $p<0.0001$ ), had a significantly higher proportion of premature responses (risk preference: $F_{(1,25)}=6.76, p=0.02$ ), and exhibited shorter latencies to collect reward (risk preference: $\left.F_{(1,25)}=14.67, p=0.001\right)$.

The risk-preferring and optimal rats in the IOFC and PrL cohorts exhibited slightly different choice patterns (choice $\times$ brain region $\times$ risk preference: $\left.F_{(2,51)}=5.11, p=0.009\right)$. This was particularly evident in optimal rats; risk-preferring rats exhibited only a trending difference in choice preference [risk-preferring rats: choice $\times$ brain region: $F_{(2,17)}=3.45, p=0.07$ (Fig. $3 A$ ); optimal rats: choice $\times$ brain region: $F_{(3,38)}=3.99, p=0.02$ (Fig. 3B)]. Optimal rats in the PrL group chose P2 at a significantly higher rate than rats in the IOFC group $\left(t_{(12)}=2.92, p=0.01\right)$.

To assess whether damage associated with cannula implantation and the microinfusion procedure impacted decision-making on the rGT, four presurgery sessions were binned and compared with baseline data collected between infusion days. No effect was observed on P1-P4

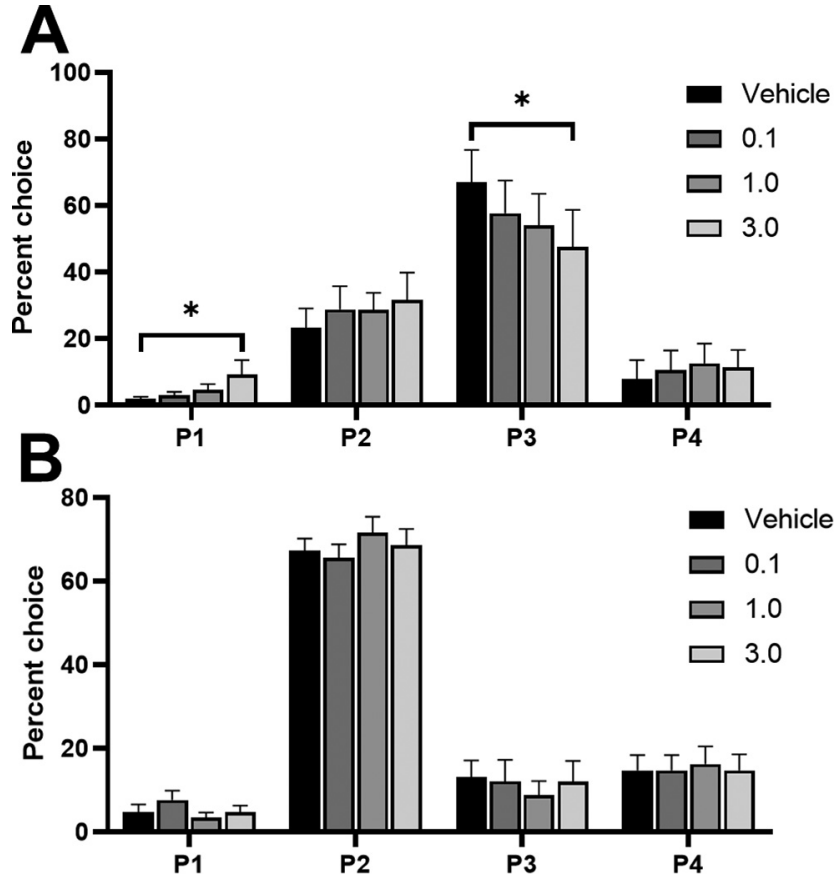

Figure 4. Effects of intra-IOFC infusions of the $5-\mathrm{HT}_{2 \mathrm{C}}$ receptor agonist RS 102221 in $(\boldsymbol{A})$ risk-preferring animals $(n=9)$ and $(\boldsymbol{B})$ optimal decision-makers $(n=9)$ on P1-P4 choice. Antagonism of the $5-\mathrm{HT}_{2 \mathrm{C}}$ receptor in the IOFC decreased $\mathrm{P} 3$ choice and increased $\mathrm{P} 1$ choice on the crGT in risk-preferring rats. Data are expressed as mean \pm SEM; ${ }^{*} p<0.05$ compared with vehicle.

choice, indicating that procedure-associated damage did not significantly impact their decision-making (data bin $\times$ choice: $\left.F_{(3,66)}=0.40, p=0.75\right)$.

\section{Microinfusions into IOFC \\ Choice}

We observed a significant shift in choice when comparing all doses in an omnibus ANOVA, that was dependent on the rats' choice patterns at baseline (dose $\times$ choice $\times$ risk status: $\left.F_{(7,117)}=7.34, p=0.04\right)$. This effect was only present in risk-preferring rats [dose $\times$ choice, risk-preferring: $F_{(9,72)}=3.09, p=0.003$ (Fig. $4 A$ ); optimal: $F_{(9,72)}=$ $0.01, p=0.30$ (Fig. 4B)]. Post hoc analyses revealed a significant reduction in P3 choice $\left(t_{(8)}=2.49, p=0.04\right)$ and a significant increase in $\mathrm{P} 1$ choice $\left(t_{(8)}=-2.74, p=0.03\right)$ in risk-preferring rats, when comparing vehicle to the highest dose. This resulted in a significant increase in risk score in these rats (dose: $F_{(3,24)}=4.39, p=0.01$; vehicle vs $\left.1.0 \mu \mathrm{g}: t_{(8)}=-2.53, p=0.04\right)$. No effect was observed on $\mathrm{P} 2$ or $\mathrm{P} 4$ choice $\left(\mathrm{P} 2: t_{(8)}=-1.52, p=0.17 ; \mathrm{P} 4: t_{(8)}=-1.59\right.$, $p=0.15)$.

\section{Premature responding and other variables}

No effect on premature responding was observed (dose: $F_{(3,48)}=1.10, p=0.36$ ). A significant shift in omissions was evident in optimal rats only (dose $\times$ risk status: $F_{(3,38)}=3.66, p=0.03$; dose, optimal: $F_{(3,21)}=3.56, p=$ 0.03 ; risk-preferring: $\left.F_{(1,11)}=1.20, p=0.32\right)$. Post hoc analyses revealed a significant reduction in omitted trials 
Table 1: Mean values of all other task variables (\% premature response, choice latency, collect latency, omissions, trials completed) at each dose of RS 102221 delivered into either the IOFC or PrL

\begin{tabular}{lllllll}
\hline Region & Dose & \% Premature responses & Choice latency & Collect latency & Omissions & Trials completed \\
\hline IOFC & 0 & $28.62 \pm 4.05$ & $1.28 \pm 0.19$ & $1.32 \pm 0.07$ & $1.50 \pm 0.53$ & $75.38 \pm 5.28$ \\
& 0.1 & $29.66 \pm 3.65$ & $1.20 \pm 0.12$ & $1.19 \pm 0.04$ & $0.94 \pm 0.22$ & $77.56 \pm 5.11$ \\
& 1.0 & $31.90 \pm 4.71$ & $1.22 \pm 0.12$ & $1.24 \pm 0.03$ & $0.39 \pm 0.18$ & $75.63 \pm 5.05$ \\
& 3.0 & $25.92 \pm 3.72$ & $1.30 \pm 0.13$ & $2.01 \pm 0.42$ & $0.94 \pm 0.51$ & $83.63 \pm 6.41$ \\
PrL & 0 & $34.05 \pm 5.35$ & $1.13 \pm 0.12$ & $1.42 \pm 0.16$ & $1.36 \pm 0.72$ & $69.98 \pm 7.94$ \\
& 0.1 & $31.94 \pm 6.59$ & $1.14 \pm 0.12$ & $1.29 \pm 0.05$ & $0.91 \pm 0.44$ & $73.37 \pm 9.67$ \\
& 1.0 & $28.64 \pm 6.30$ & $1.31 \pm 0.17$ & $1.21 \pm 0.07$ & $1.18 \pm 0.46$ & $75.41 \pm 10.12$ \\
& 3.0 & $24.19 \pm 5.00$ & $1.34 \pm 0.15$ & $1.24 \pm 0.12$ & $0.91 \pm 0.44$ & $78.66 \pm 10.78$ \\
\hline
\end{tabular}

Data are mean \pm SEM.

in these rats, when comparing vehicle to the highest dose $\left(t_{(8)}=2.40, p=0.04\right)$. However, these rats showed a significantly higher level of omissions at vehicle compared with their baseline data $\left(t_{(8)}=2.41, p=0.04\right)$. No effect was observed on latency variables or trials completed (all $F<1.33 ; p>0.27$; Table 1).

\section{Reinforcer devaluation Choice}

Behavioral data from devaluation sessions for each group of rats (naive, vehicle, or drug) was compared with their baseline data (naive group: no manipulation; vehicle group: vehicle dosing data; drug group: highest RS 102221 dose data). In Figure 5A, choice of the P1-P4 options is depicted as a difference in \% choice between baseline and devaluation sessions (baseline subtracted from devaluation) for each group. This was done to highlight shifts in choice separate from overall cohort differences in the selection of the different options. Mean values and SEMs for each session and group can be found in Table 2. Risky and optimal rats are grouped together as statistical analyses did not reveal any effects that were dependent on risk status. We observed a significant shift in P1-P4 choice in response to reinforcer devaluation that was dependent on group but not risk status (devaluation $\times$ choice $\times$ group: $F_{(9,57)}=2.56, p=0.02$; Fig. $5 A$ ). Naive rats did not demonstrate a shift in their choice profile (devaluation $\times$ choice: $\left.F_{(3,36)}=1.58, p=0.21\right)$. Similarly, we did not observe any change in the choice profile of rats that received a vehicle dose in addition to devaluation, versus vehicle alone (devaluation $\times$ choice: $\left.F_{(3,9)}=1.39, p=0.31\right)$. Conversely, rats in the drug + devaluation condition exhibited a shift in their decision-making that was significantly different from the effect of the drug alone (devaluation $\times$ choice: $F_{(3,12)}=5.64$, $p=0.01$ ). When comparing P1-P4 choice between the drug versus drug + devaluation condition with post hoc analyses, we observed a trending reduction in P2 choice $\left(t_{(5)}=2.35\right.$, $p=0.07)$ and a significant increase in P4 choice $\left(t_{(5)}=-3.76\right.$, $p=0.01$ ). A comparison of the drug + devaluation versus vehicle condition in these rats reached marginal significance (devaluation $\times$ choice: $F_{(2,6)}=3.80, p=0.08$ ), resulting from a significant increase in $\mathrm{P} 1$ and $\mathrm{P} 4$ choice $\left(\mathrm{P} 1: t_{(5)}=-4.92\right.$, $\left.p=0.004 ; \mathrm{P} 4: t_{(5)}=-5.95, p=0.002\right)$. Thus, rats who received an infusion of the highest dose of RS 102221 into the IOFC were uniquely sensitive to the effects of reinforcer devaluation on choice, exhibiting a choice profile that differed from their decision-making patterns after receiving either vehicle or drug alone.

\section{Other task variables}

We observed a significant shift in trials completed that was dependent on group (group $\times$ devaluation: $F_{(3,19)}=$ $6.25, p=0.004$; Fig. $5 B$ ). Only rats that received intraIOFC RS 102221 completed significantly fewer trials in response to devaluation (drug: $F_{(1,4)}=24.12, p=0.008$; vehicle: $F_{(1,3)}=4.64, p=0.12$; naive: $\left.F_{(1,14)}=2.50, p=0.14\right)$. Rats in all groups exhibited decreased premature responding $\left(F_{(1,19)}=39.37, p<0.0001\right.$; Fig. 5C) and increased latencies to choose an option (devaluation: $F_{(1,19)}=17.52, p=0.001$; Fig. $\left.5 D\right)$ in response to reinforcer devaluation. No effect was observed on omissions or latencies to collect reward (all $F<1.16$, all $p>0.30$; Fig. $5 E)$. See Table 3 for the mean values and SEMs for the reported variables in each group and session.

\section{Microinfusions into PrL region Choice}

When examining choice in an omnibus ANOVA, we observed a significant effect of dose that did not interact with risk status or the different options (dose: $F_{(3,27)}=$ $3.39, p=0.03$ ), potentially indicative of increased variability or noise in rats' response patterns that does not reliably load on one option or another. Figure $6 A, B$ depicts the $\%$ choice of each option at each dose in risk-preferring and optimal rats, respectively. Comparing the choice of P1P4 between vehicle and each dose did not reveal any significant effects (all $t<1.99$, all $p>0.09$ ). Correspondingly, there was no significant effect on risk score (dose: $\left.F_{(3,27)}=0.64, p=0.60\right)$. These results indicate that while rats' decision-making patterns became more variable across doses, there was no clear pattern of an increase or decrease of choice for any particular option.

\section{Premature responding and other variables}

No significant effect was observed on premature responding, latency variables, omissions, or trials completed (all $F<1.68$; $p>0.20$; see Table 1 for mean values and SEMs of task variables at each dose).

\section{Discussion}

Results from this study demonstrated that infusing a 5$\mathrm{HT}_{2 \mathrm{C}}$ antagonist directly into the IOFC, but not the PrL 


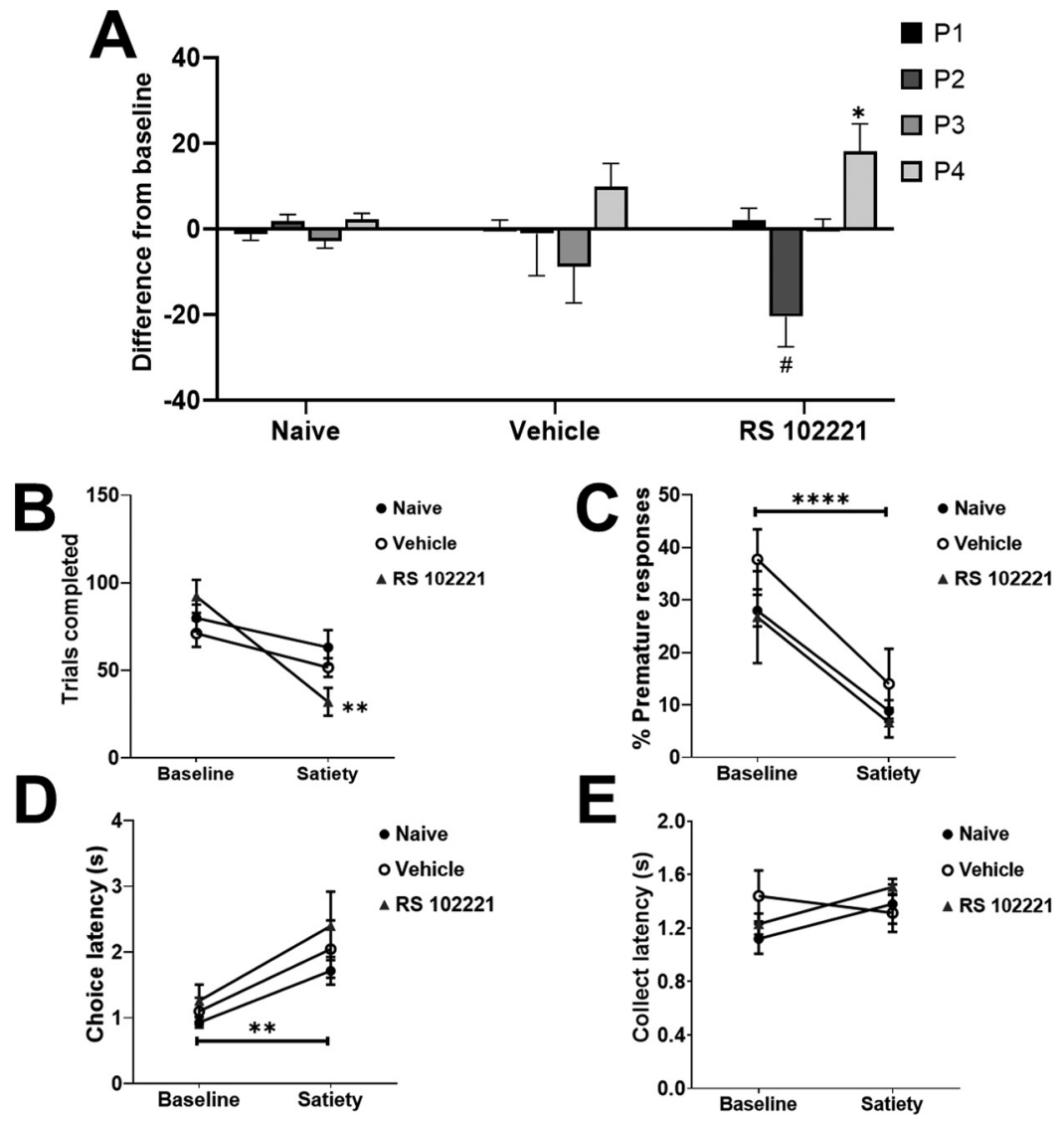

Figure 5. Effects of sucrose pellet devaluation on choice preference and other task variables. Only rats receiving intra-IOFC RS 102221 shifted their choice profile $(\boldsymbol{A})$ and completed significantly fewer trials $(\boldsymbol{B})$ in response to devaluation. Animals in all groups exhibited a significant reduction in premature responding $(\boldsymbol{C})$ and increased choice latencies $(\boldsymbol{D})$. No shift in latencies to collect reward were observed in any group $(\boldsymbol{E})$. In panel $\boldsymbol{A}$, data are expressed as the mean change in \% choice from baseline \pm SEM to illustrate effects independent of differences in preference for each option between cohorts. Data are expressed as mean \pm SEM in all other panels; ${ }^{*} p<0.05,{ }^{* *} p<0.01,{ }^{* * * *} p<0.0001, \# p=0.07$ compared with each group's own baseline.

region, improved decision-making in risk-preferring rats without negatively impacting impulsivity measures. 5$\mathrm{HT}_{2 \mathrm{C}}$ antagonism in the IOFC also restored behavioral sensitivity to reinforcer devaluation, indicating that flexibility in reward valuation was increased by this manipulation. In contrast, intra-PrL infusions of RS 102221 did not increase optimal choice. The ability of $5-\mathrm{HT}_{2 \mathrm{C}}$ antagonism to ameliorate cue-enhanced risky choice therefore exhibits at least some regional specificity within the frontal cortices.
While the damage associated with surgeries and infusions is typical of studies using this technique, we cannot rule out that this may have altered the functioning of prefrontal circuitry and influenced the observed results. Nevertheless, within-subjects comparisons to vehicle dosing, along with counterbalanced orders of doses among the rats, supports the conclusion that the effect of $5-\mathrm{HT}_{2 \mathrm{C}}$ antagonism in the IOFC is not simply because of procedure-associated damage. Furthermore, choice patterns during baseline sessions did not shift throughout the

Table 2: Percent choice of the different options in response to acute sucrose pellet devaluation in surgically-naive rats, rats who received intra-IOFC RS 102221 in addition to devaluation, and rats who received intra-IOFC vehicle and devaluation

\begin{tabular}{llllll}
\hline Group & Condition & P1 & P2 & P3 & P4 \\
\hline Surgically naive & Baseline & $4.82 \pm 1.33$ & $51.72 \pm 9.17$ & $24.93 \pm 8.68$ & $18.53 \pm 7.18$ \\
Drug + devaluation & Devaluation & $3.62 \pm 1.33$ & $53.50 \pm 9.25$ & $22.09 \pm 8.12$ & $20.79 \pm 6.66$ \\
& Vehicle & $1.59 \pm 0.79$ & $56.57 \pm 7.93$ & $25.91 \pm 11.55$ & $15.93 \pm 8.51$ \\
& Drug & $7.13 \pm 1.67$ & $58.16 \pm 5.44$ & $18.67 \pm 8.12$ & $16.04 \pm 6.56$ \\
Vehicle + devaluation & Devaluation & $9.29 \pm 1.73$ & $37.67 \pm 10.06$ & $18.94 \pm 8.34$ & $34.10 \pm 12.09$ \\
& Vehicle & $4.83 \pm 2.46$ & $41.01 \pm 13.12$ & $40.03 \pm 19.23$ & $14.13 \pm 5.56$ \\
& Devaluation & $4.92 \pm 1.72$ & $39.91 \pm 10.92$ & $31.19 \pm 17.17$ & $23.98 \pm 9.85$
\end{tabular}

Data are mean \pm SEM. 
Table 3: Mean values of all other task variables (\% premature response, choice latency, collect latency, omissions, trials completed) in response to acute sucrose pellet devaluation in surgically-naive rats, rats who received intra-IOFC RS 102221 in addition to devaluation, and rats who received intra-IOFC vehicle and devaluation

\begin{tabular}{llrllll}
\hline Group & Condition & \% Premature responses & Choice latency & Collect latency & Omissions & Trials completed \\
\hline Surgically naive & Baseline & $27.96 \pm 3.00$ & $0.93 \pm 0.08$ & $1.12 \pm 0.11$ & $0.25 \pm 0.11$ & $79.89 \pm 7.63$ \\
& Devaluation & $8.82 \pm 2.09$ & $1.71 \pm 0.21$ & $1.38 \pm 0.15$ & $1.31 \pm 0.55$ & $63.14 \pm 9.87$ \\
Drug + devaluation & Vehicle & $26.72 \pm 5.72$ & $1.35 \pm 1.20$ & $1.57 \pm 0.35$ & $1.50 \pm 1.11$ & $80.87 \pm 11.71$ \\
& Drug & $19.96 \pm 6.94$ & $1.26 \pm 0.25$ & $1.23 \pm 0.08$ & $0.50 \pm 0.34$ & $92.18 \pm 9.46$ \\
& Devaluation & $6.61 \pm 2.81$ & $2.40 \pm 0.52$ & $1.51 \pm 0.06$ & $1.33 \pm 0.49$ & $32.00 \pm 7.93$ \\
Vehicle + devaluation & Vehicle & $37.74 \pm 5.72$ & $1.10 \pm 0.21$ & $1.33 \pm 0.19$ & $1.20 \pm 0.97$ & $71.06 \pm 7.63$ \\
& Devaluation & $14.00 \pm 6.68$ & $2.04 \pm 0.43$ & $1.31 \pm 0.14$ & $0.80 \pm 0.37$ & $51.62 \pm 5.40$ \\
\hline
\end{tabular}

Data are mean \pm SEM.

infusion procedure and were not significantly different from data collected before surgery.

Although the ratio of $5-\mathrm{HT}_{2 \mathrm{~A}}$ to $5-\mathrm{HT}_{2 \mathrm{C}}$ receptors in the $\mathrm{mPFC}$ is positively correlated with levels of premature responding on a simplified version of the 5CSRT (Anastasio et al., 2015), we did not see any increase in premature responding when RS 102221 was delivered into the PrL, similar to a previous report (Robinson et al., 2008). There were fewer rats in the PrL cohort $(n=11)$ than the IOFC cohort $(n=18)$, but the sample size for both experiments was certainly comparable to other studies using this technique. Considerable data suggest an association between $5-\mathrm{HT}_{2 \mathrm{C}}$ receptor activity in the mPFC, motor impulsivity, and relapse-like cocaine-seeking in rats (Filip and Cunningham, 2003; Anastasio et al., 2014; Swinford-Jackson et al., 2016). Furthermore, increased

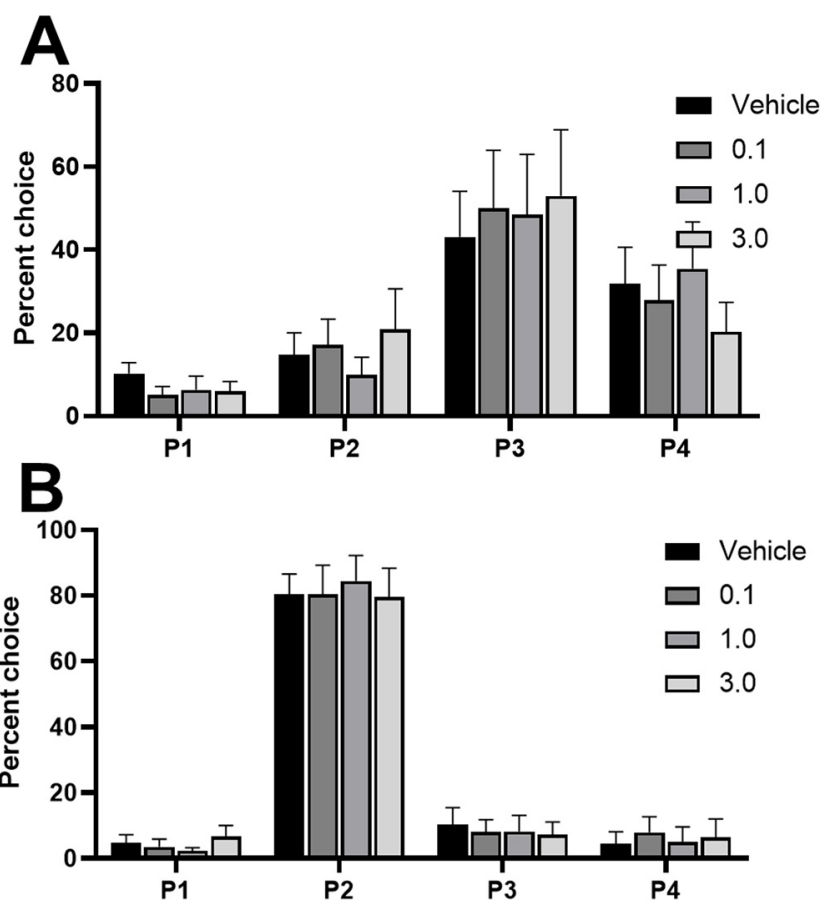

Figure 6. Effects of intra-PrL infusions of the $5-\mathrm{HT}_{2 \mathrm{C}}$ receptor agonist RS 102221 in $(\boldsymbol{A})$ risk-preferring animals $(n=5)$ and $(\boldsymbol{B})$ optimal decision-makers $(n=6)$ on P1-P4 choice. Antagonism of the $5-\mathrm{HT}_{2 \mathrm{C}}$ receptor in the PrL did not significantly increase or decrease choice of any particular option in either risk-preferring $(\boldsymbol{A})$ or optimal $(\boldsymbol{B})$ rats. Data are expressed as mean \pm SEM. risky decision-making following cocaine self-administration correlates with at least one measure of relapse vulnerability (Ferland and Winstanley, 2017). Given that both greater risky choice and higher levels of premature responding are associated with behavioral markers of cocaine addiction in rats and are significantly correlated at the population level (Barrus et al., 2015), we might expect a greater overlap in the neurobiological control of these cognitive processes. However, numerous studies now show that these phenomena are subject to differential neural and pharmacological regulation (Zeeb et al., 2009; Barrus and Winstanley, 2016; Adams et al., 2017; Betts et al., 2021; Chernoff et al., 2021). While impulsive action and risky decision-making may interact synergistically in the manifestation of impulse control and addiction disorders, they ultimately may represent somewhat dissociable pathways to addiction.

In contrast to the effects of systemic administration, which impacted both optimal and risk-preferring rats, intra-IOFC administration of a $5-\mathrm{HT}_{2 \mathrm{C}}$ antagonist only improved decision-making in those that exhibited a preference for the risky options at baseline. The neural locus whereby $5-\mathrm{HT}_{2 \mathrm{C}}$ antagonism further optimizes choice in animals already making advantageous decisions remains to be determined, but is clearly neither the IOFC nor the PrL. The selective effects of drug infusions in risk-preferring rats also suggests that the neural architecture underlying the decision-making process in optimal versus risky rats differs, either in the regions forming the network, the weight given to the output of those regions in guiding choice, or the computational analyses performed by key nodes. Indeed, data across species support the view that individual differences in choice preference can be attributed to differential activity across brain networks. Cues present in the environment can also influence the adoption of a behavioral strategy; the considerable literature on sign-trackers versus goal-trackers may best exemplify the significant individual differences in how such cues can be used to guide behavior (Flagel et al., 2009, 2011; Saunders and Robinson, 2010, 2013). Illuminating a cue light during lengthy delays between response and reward delivery can decrease delay discounting (i.e., reduction in a reward's subjective value because of waiting period) in rats (Cardinal et al., 2000). However, the presence of this cue does not eliminate the large individual differences in animals' preferences for smaller-sooner versus largerlater rewards. Inactivation of the IOFC only reduced 
choice of the larger-later reward in rats that showed a high baseline preference for this option; an effect that was also observed following local infusions of dopamine antagonists (Zeeb et al., 2010). Thus, the hypothesis that recruitment of the IOFC into the decision-making process depends on the presence of cues, and that decision-making patterns are only IOFC-dependent in a subpopulation of individuals that use those cues to guide behavior, has some precedent.

It is interesting to note that the effect in risk-preferring animals was specific to a decrease in P3 (their preferred risky option) and an increase in $\mathrm{P} 1$, the optimal option offering the most frequent wins but the smallest reward size (one sucrose pellet). This could be because of either an increased sensitivity to the length and/or frequency of timeout penalties, or increased impact of frequent winning trials. This may help explain the specific effect observed in risk-preferring animals; for optimal rats, the difference between P1 and P2 in time-out penalty length/frequency and reward frequency may not be large enough to shift decision-making patterns away from their preferred option. Investigating neural activity following rewards and time-out penalties on each option within the IOFC could shed light on these hypotheses.

Indeed, identifying how audiovisual cues modulate IOFC neuronal firing as well as the impact of $5-\mathrm{HT}_{2 \mathrm{C}}$ antagonism are important next steps. The $5-\mathrm{HT}_{2 \mathrm{C}}$ receptor is an excitatory GPCR found throughout the rat central nervous system (Clemett et al., 2000). Previous studies have demonstrated that $5-\mathrm{HT}_{2 \mathrm{C}}$ receptors are primarily located in the deep layers of the PFC in rats (Pompeiano et al., 1994; Liu et al., 2007). In the mPFC, at least $50 \%$ of receptors are localized to GABAergic interneurons, and are hypothesized to regulate the output of pyramidal cells (Liu et al., 2007). Considerably less is known about the localization and function of $5-\mathrm{HT}_{2 \mathrm{C}}$ receptors in the OFC. Interneurons in this region play a key role in reversal learning, so it is possible that modulation of GABAergic interneuron activity by the $5-\mathrm{HT}_{2 \mathrm{C}}$ antagonist may drive the improvement in choice seen here (Bissonette et al., 2015).

As noted in the introduction, previous literature has strongly implicated the $5-\mathrm{HT}_{2 \mathrm{C}}$ receptor in cue-mediated behaviors such as cue-induced cocaine seeking and responding for a conditioned reinforcer through its regulation of the mesolimbic dopamine system (Pentkowski et al., 2010; Browne et al., 2017). Based on this work, 5$\mathrm{HT}_{2 \mathrm{C}}$ receptor antagonists should increase motor impulsivity and potentiate the risk-promoting effect of cues in the rGT. While the former is true, the latter is clearly not. $5-\mathrm{HT}_{2 \mathrm{C}}$ receptor antagonism must therefore alter decision-making through an alternate, yet concurrent mechanism. The current data implicate the IOFC as one critical locus, and an increase in behavioral flexibility as a potential cognitive mechanism, through which decision-making may improve. Serotonergic activity within the OFC, and the $2 \mathrm{C}$ receptor, have been implicated in cognitive flexibility by multiple previous studies (Boulougouris and Robbins, 2010; Alsiö et al., 2015, 2021; Barlow et al., 2015). Previous results have indicated that circuitry between the OFC and basolateral amygdala (BLA) supports shifts in choice following reinforcer devaluation on the uncued rGT (Zeeb and Winstanley, 2013). It may be that this circuitry is also involved in cue-induced inflexibility on the task. This pathway certainly plays a role in cue-based decision-making, as BLA-OFC projections are essential for guiding decision-making based on cue-triggered reward representations (Lichtenberg et al., 2017).

It is notable that decision-making on the cued task was not altered by reinforcer devaluation in naive or vehicletreated rats, in contrast to the effects of this manipulation reported previously in the absence of the cues (Zeeb and Winstanley, 2013). Decision-making in other tasks that require considerably more sessions to train remain sensitive to changes in outcome value, indicating that simple repetition of actions in complex cognitive tasks is not sufficient to produce habitual behavior through procedural motor learning (Cocker et al., 2012). Indeed, goal-directed control can be maintained following prolonged training even if automatization of certain action sequences occurs (Garr and Delamater, 2019). Acute satiety with regular chow did not shift choice patterns on the uncued rGT and thus the effect of reinforcer devaluation on choice can be attributed to shifts in goal-directed action rather than reduced motivation. While acute satiety with chow has not been tested on the crGT, it stands to reason that reduced motivation alone would similarly leave choice patterns unaffected. As such, decision-making on the crGT may fail one of the critical tests of true goal-directed behavior, in that it is insensitive to changes in the goal's value (Balleine and Dickinson, 1992). If this is the case, then 5$\mathrm{HT}_{2 \mathrm{C}}$ antagonism in the IOFC may shift rats toward a goal-directed response strategy and therefore restore sensitivity to reinforcer value.

Regardless of experimental condition, motivation to engage in the task declined; premature responses decreased when animals were sated, while the latency to choose an option increased. Interestingly, while latency to collect reward is sensitive to satiety on the uncued rGT (Zeeb and Winstanley, 2013), this measure did not significantly increase with reinforcer devaluation in any group. Furthermore, the inclusion of cues on the rGT results in decreased collection latencies, particularly in risky rats (Hathaway et al., 2021). It therefore may be that the presence of the cues invigorates responding to reward delivery, and the effect is not dependent on the value of the reward or computation within the IOFC, as this measure was unaffected by devaluation with or without drug administration. In addition, the number of trials completed decreased in all rats in response to devaluation, but the effect only reached significance in rats who also received $R S$ 102221. On the uncued rGT, trial completion was reduced in response to devaluation, but this was prevented by disconnecting the BLA and IOFC (Zeeb and Winstanley, 2013). Thus, the IOFC may play a key role in inhibiting perseverative responding on the rGT. That intra-IOFC infusion of RS 102221 selectively rendered decision-making and trial completion more sensitive to reinforcer devaluation, without altering the effects of satiety on other variables, further supports the hypothesis that local $5-\mathrm{HT}_{2 \mathrm{C}}$ antagonism facilitated some form of cognitive flexibility. 
As alluded to above, if changes to the value of the reinforcer do not alter behavior, that behavior is thought to be under habitual rather than goal-directed control. However, it could be that the behavior in question is instead reinforced by another aspect of the environment. Given that the cues are concurrent with reward delivery, it could be argued that "reward + cue" has formed a compound reinforcer. Selective devaluation of only one component of this reinforcer may therefore fail to alter response patterns. Certainly, in the drug addiction literature, cues that are present when drug is taken acquire incentive motivational salience that does not decline even when users do not obtain pleasure from drug ingestion (Robinson and Berridge, 1993). However, this is believed to be a highly aberrant state, driven by supraphysiological drug-induced dopamine release amplifying associative learning between cues and drugs. Why sound and light cues should exert the same effect in the current task is unclear, given that rats typically show a dramatic reduction in responding for reward-paired cues following devaluation of that reward (Hatfield et al., 1996; Pickens et al., 2005; McDannald et al., 2014). This is not the first time that similarities have been observed between responding on the crGT and responding for addictive drugs, as there is some evidence that risky choice on-task and cocaine self-administration may cross-sensitize and/or substitute for one another (Ferland et al., 2019; Hynes et al., 2021). However, systemic $5-\mathrm{HT}_{2 \mathrm{C}}$ antagonism increases responding for drug (Fletcher et al., 2002), yet decreases risky choice here, indicating that the pharmacological regulation of these processes is not uniform.

Instead of the cues acquiring incentive motivational properties that are now independent of reward value, another alternative is that these highly salient audiovisual cues overshadow sucrose pellet delivery to some extent (Pavlov, 1927), such that a change in the sucrose pellet value does not dominate behavior in the presence of the cues. To our knowledge, neither the IOFC nor $5-\mathrm{HT}_{2 \mathrm{C}}$ receptor signaling have been evaluated in overshadowing experiments, and as such further discussion of this hypothesis is premature. If overshadowing is taking place, then the attenuation of learning about the devalued state of the reward is highly specific to the decision-making process, as devaluation still impacted latencies and motor impulsivity, indicating a reduction in the ability of the sucrose pellet rewards to motivate and invigorate behavior.

Given that $5-\mathrm{HT}_{2 \mathrm{C}}$ receptor antagonism can clearly increase incentive motivation for rewards and rewardpaired cues, likely through actions in the mesolimbic dopamine pathway, the following question remains: why does this mechanism not dominate the decision-making process? One potential answer comes from human literature examining the impact of serotonin depletion on model-based behavior: goal-directed choice is impaired when learning from rewards but enhanced when learning from punishments (Worbe et al., 2016). Computational modeling analyses have revealed that the addition of cues to the rGT specifically impairs learning from the time-out penalties (Langdon et al., 2019). As such, manipulating serotonergic activity within the IOFC may promote the correct integration of punishments into the stored actionoutcome contingencies for risk-preferring rats, rather than influencing the incentive motivation of the cues. This is in line with the finding that responding for a conditioned reinforcer does not correlate with risky choice on the crGT, at least in female rats (Winstanley and Tremblay, 2016), indicating that incentive motivation is not primarily responsible for cue-induced risky choice on this task. The activity of striatal neurons, although influenced by neuromodulators like dopamine, is still primarily driven by cortical inputs. It is therefore possible that $5-\mathrm{HT}_{2 \mathrm{C}}$-receptor mediated modulation of IOFC output is sufficient to dominate the behavioral response.

Overall, these results indicate that $5-\mathrm{HT}_{2 \mathrm{C}}$ antagonism in the IOFC can ameliorate cue-induced disadvantageous risky choice in rats with preexisting preferences for these risky options. The lack of effect in optimal rats, together with recent computational modeling analyses, suggests there are underlying differences in the processing or storage of action-outcome contingencies between optimal and risk-preferring animals. It is currently unknown whether this is because of distinct activity patterns within the IOFC or differential involvement of downstream targets. Future studies could examine activity in the IOFC during crGT learning and performance in risk-preferring versus optimal rats to address this question.

Furthermore, these results suggest that targeting flexibility may be a viable approach to improving decisionmaking in individuals with impaired cost/benefit decisionmaking, specifically in the presence of cues. This would have implications for the treatment of behavioral addictions and substance use disorders, in which individuals show marked impairments in disadvantageous risky decision-making and processing of reward-associated cues (Goudriaan et al., 2005; Limbrick-Oldfield et al., 2017; Zilberman et al., 2019). Recent clinical trials for the 5$\mathrm{HT}_{2 \mathrm{C}}$ agonist in the treatment of substance use disorder have been unsuccessful. Results from these studies indicate the necessity to attend to individual differences, as only rats with preexisting deficits in decision-making show improvements in response to the antagonist. Greater specificity of targeting to regions may also improve treatment, as the antagonist can increase impulsivity via other pathways. Allosteric modulators of the 5$\mathrm{HT}_{2 \mathrm{C}}$ receptor may be worth pursuing in this regard. Given that numerous effective psychoactive medications act on the serotonin system, there is every reason to feel cautiously optimistic that a viable serotonergic medication could be developed for disorders hallmarked or exacerbated by risky decision-making.

\section{References}

Adams WK, Barkus C, Ferland JN, Sharp T, Winstanley CA (2017) Pharmacological evidence that $5-\mathrm{HT} 2 \mathrm{C}$ receptor blockade selectively improves decision making when rewards are paired with audiovisual cues in a rat gambling task. Psychopharmacology (Berl) 234:3091-3104.

Alsiö J, Nilsson SR, Gastambide F, Wang RA, Dam SA, Mar AC, Tricklebank M, Robbins TW (2015) The role of 5-HT2C receptors in 
touchscreen visual reversal learning in the rat: a cross-site study. Psychopharmacology (Berl) 232:4017-4031.

Alsiö J, Lehmann O, McKenzie C, Theobald DE, Searle L, Xia J, Dalley J, Robbins TW (2021) Serotonergic innervations of the orbitofrontal and medial-prefrontal cortices are differentially involved in visual discrimination and reversal learning in rats. Cereb Cortex 31:1090-1105.

Amodeo LR, McMurray MS, Roitman JD (2017) Orbitofrontal cortex reflects changes in response-outcome contingencies during probabilistic reversal learning. Neuroscience 345:27-37.

Anastasio NC, Stutz SJ, Fox RG, Sears RM, Emeson RB, DiLeone RJ, O'Neil RT, Fink LH, Li D, Green TA, Moeller FG, Cunningham KA (2014) Functional status of the serotonin 5-HT2C receptor (5HT2CR) drives interlocked phenotypes that precipitate relapse-like behaviors in cocaine dependence. Neuropsychopharmacology 39:370-382.

Anastasio NC, Stutz SJ, Fink LHL, Swinford-Jackson SE, Sears RM, DiLeone RJ, Rice KC, Moeller FG, Cunningham KA (2015) Serotonin (5-HT) 5-HT2A receptor (5-HT2AR):5-HT2CR imbalance in medial prefrontal cortex associates with motor impulsivity. ACS Chem Neurosci 6:1248-1258.

Balleine B, Dickinson A (1992) Signalling and incentive processes in instrumental reinforcer devaluation. Q J Exp Psychol B 45:285301.

Barlow RL, Alsiö J, Jupp B, Rabinovich R, Shrestha S, Roberts AC, Robbins TW, Dalley JW (2015) Markers of serotonergic function in the orbitofrontal cortex and dorsal raphé nucleus predict individual variation in spatial-discrimination serial reversal learning. Neuropsychopharmacology 40:1619-1630.

Barrus MM, Winstanley CA (2016) Dopamine D3 receptors modulate the ability of win-paired cues to increase risky choice in a rat gambling task. J Neurosci 36:785-794.

Barrus MM, Hosking JG, Zeeb FD, Tremblay M, Winstanley CA (2015) Disadvantageous decision-making on a rodent gambling task is associated with increased motor impulsivity in a population of male rats. J Psychiatry Neurosci 40:108-117.

Baxter MG, Parker A, Lindner CC, Izquierdo AD, Murray EA (2000) Control of response selection by reinforcer value requires interaction of amygdala and orbital prefrontal cortex. J Neurosci 20:4311-4319.

Bechara A, Damasio AR, Damasio H, Anderson SW (1994) Insensitivity to future consequences following damage to human prefrontal cortex. Cognition 50:7-15.

Betts GD, Hynes TJ, Winstanley CA (2021) Pharmacological evidence of a cholinergic contribution to elevated impulsivity and risky decision making caused by adding win-paired cues to a rat gambling task. J Psychopharmacol 35:701-712.

Bissonette GB, Schoenbaum G, Roesch MR, Powell EM (2015) Interneurons are necessary for coordinated activity during reversal learning in orbitofrontal cortex. Biol Psychiatry 77:454-464.

Bolla KI, Eldreth DA, London ED, Kiehl KA, Mouratidis M, Contoreggi C, Matochik JA, Kurian V, Cadet JL, Kimes AS, Funderburk FR, Ernst M (2003) Orbitofrontal cortex dysfunction in abstinent cocaine abusers performing a decision-making task. Neuroimage 19:1085-1094.

Boulougouris V, Robbins TW (2010) Enhancement of spatial reversal learning by $5-\mathrm{HT} 2 \mathrm{C}$ receptor antagonism is neuroanatomically specific. J Neurosci 30:930-938.

Browne CJ, Ji X, Higgins GA, Fletcher PJ, Harvey-Lewis C (2017) Pharmacological modulation of $5-\mathrm{HT} 2 \mathrm{C}$ receptor activity produces bidirectional changes in locomotor activity, responding for a conditioned reinforcer, and mesolimbic DA release in C57BL/6 mice. Neuropsychopharmacology 42:2178-2187.

Cardinal RN, Robbins TW, Everitt BJ (2000) The effects of d-amphetamine, chlordiazepoxide, alpha-flupenthixol and behavioural manipulations on choice of signalled and unsignalled delayed reinforcement in rats. Psychopharmacology (Berl) 152:362-375.

Carli M, Robbins TW, Evenden JL, Everitt BJ (1983) Effects of lesions to ascending noradrenergic neurones on performance of a 5choice serial reaction task in rats; implications for theories of dorsal noradrenergic bundle function based on selective attention and arousal. Behav Brain Res 9:361-380.

Cherkasova MV, Clark L, Barton JJS, Schulzer M, Shafiee M, Kingstone A, Stoessl AJ, Winstanley CA (2018) Win-concurrent sensory cues can promote riskier choice. J Neurosci 38:1036210370.

Chernoff CS, Hynes TJ, Winstanley CA (2021) Noradrenergic contributions to cue-driven risk-taking and impulsivity. Psychopharmacology (Berl) 238:1765-1779.

Clemett D, Punhani T, Duxon MS, Blackburn TP, Fone KCF (2000) Immunohistochemical localisation of the $5-\mathrm{HT} 2 \mathrm{C}$ receptor protein in the rat CNS. Neuropharmacology 39:123-132.

Cocker PJ, Hosking JG, Benoit J, Winstanley CA (2012) Sensitivity to cognitive effort mediates psychostimulant effects on a novel rodent cost/benefit decision-making task. Neuropsychopharmacology 37:1825-1837.

Economidou D, Theobald DEH, Robbins TW, Everitt BJ, Dalley JW (2012) Norepinephrine and dopamine modulate impulsivity on the five-choice serial reaction time task through opponent actions in the shell and core sub-regions of the nucleus accumbens. Neuropsychopharmacology 37:2057-2066.

Ferland JN, Winstanley CA (2017) Risk-preferring rats make worse decisions and show increased incubation of craving after cocaine self-administration. Addict biol 22:991-1001.

Ferland JN, Hynes TJ, Hounjet CD, Lindenbach D, Vonder Haar C, Adams WK, Phillips AG, Winstanley CA (2019) Prior exposure to salient win-paired cues in a rat gambling task increases sensitivity to cocaine self-administration and suppresses dopamine efflux in nucleus accumbens: support for the reward deficiency hypothesis of addiction. J Neurosci 39:1842-1854.

Filip M, Cunningham KA (2003) Hyperlocomotive and discriminative stimulus effects of cocaine are under the control of serotonin(2C) (5-HT(2C)) receptors in rat prefrontal cortex. J Pharmacol Exp Ther 306:734-743.

Flagel SB, Akil H, Robinson TE (2009) Individual differences in the attribution of incentive salience to reward-related cues: implications for addiction. Neuropharmacology 56 [Suppl 1]:139-148.

Flagel SB, Clark JJ, Robinson TE, Mayo L, Czuj A, Willuhn I, Akers CA, Clinton SM, Phillips PE, Akil H (2011) A selective role for dopamine in stimulus-reward learning. Nature 469:53-57.

Fletcher PJ, Grottick AJ, Higgins GA (2002) Differential effects of the 5-HT(2A) receptor antagonist M100907 and the 5-HT(2C) receptor antagonist SB242084 on cocaine-induced locomotor activity, cocaine self-administration and cocaine-induced reinstatement of responding. Neuropsychopharmacology 27:576-586.

Garr E, Delamater AR (2019) Exploring the relationship between actions, habits, and automaticity in an action sequence task. Learn Mem 26:128-132.

Goudriaan AE, Oosterlaan J, de Beurs E, van den Brink W (2005) Decision making in pathological gambling: a comparison between pathological gamblers, alcohol dependents, persons with Tourette syndrome, and normal controls. Brain Res Cogn Brain Res 23:137-151.

Hatfield T, Han JS, Conley M, Gallagher M, Holland P (1996) Neurotoxic lesions of basolateral, but not central, amygdala interfere with Pavlovian second-order conditioning and reinforcer devaluation effects. J Neurosci 16:5256-5265.

Hathaway BA, Hrelja KM, Harris CBW, Hynes TJ, Winstanley CA (2021) Investigating the behavioural effects of win-associated cues, outcome-associated cues, and randomly occurring cues on risky decision making. Society for Neuroscience Global Connectome: A Virtual Event. January 11-13, 2021, Virtual.

Hynes TJ, Hrelja KM, Hathaway BA, Hounjet CD, Chernoff CS, Ebsary SA, Betts GD, Russell B, Ma L, Kaur S, Winstanley CA (2021) Dopamine neurons gate the intersection of cocaine use, decision making, and impulsivity. Addict Biol 26:e13022.

Izquierdo A, Suda RK, Murray EA (2004) Bilateral orbital prefrontal cortex lesions in rhesus monkeys disrupt choices guided by both reward value and reward contingency. J Neurosci 24:7540-7548. 
Langdon AJ, Hathaway BA, Zorowitz S, Harris C, Winstanley CA (2019) Relative insensitivity to time-out punishments induced by win-paired cues in a rat gambling task. Psychopharmacology (Berl) 236:2543-2556.

Lichtenberg NT, Pennington ZT, Holley SM, Greenfield VY, Cepeda C, Levine MS, Wassum KM (2017) Basolateral amygdala to orbitofrontal cortex projections enable cue-triggered reward expectations. J Neurosci 37:8374-8384.

Limbrick-Oldfield EH, Mick I, Cocks RE, McGonigle J, Sharman SP, Goldstone AP, Stokes PR, Waldman A, Erritzoe D, Bowden-Jones $H$, Nutt D, Lingford-Hughes A, Clark L (2017) Neural substrates of cue reactivity and craving in gambling disorder. Transl Psychiatry 7:e992.

Liu S, Bubar MJ, Lanfranco MF, Hillman GR, Cunningham KA (2007) Serotonin $2 \mathrm{C}$ receptor localization in GABA neurons of the rat medial prefrontal cortex: implications for understanding the neurobiology of addiction. Neuroscience 146:1677-1688.

McDannald MA, Jones JL, Takahashi YK, Schoenbaum G (2014) Learning theory: a driving force in understanding orbitofrontal function. Neurobiol Learn Mem 108:22-27.

Pattij T, Janssen MCW, Vanderschuren LJMJ, Schoffelmeer ANM, van Gaalen MM (2007) Involvement of dopamine D1 and D2 receptors in the nucleus accumbens core and shell in inhibitory response control. Psychopharmacology (Berl) 191:587-598.

Pavlov IR (1927) Conditioned reflexes. Oxford: Oxford University Press.

Paxinos G, Watson C (1998) The rat brain in stereotaxic coordinates, Ed 4. San Diego: Academic Press.

Pentkowski NS, Duke FD, Weber SM, Pockros LA, Teer AP, Hamilton EC, Thiel KJ, Neisewander JL (2010) Stimulation of medial prefrontal cortex serotonin $2 \mathrm{C}(5-\mathrm{HT})$ receptors attenuates cocaine-seeking behavior. Neuropsychopharmacology 35:20372048.

Pickens CL, Saddoris MP, Gallagher M, Holland PC (2005) Orbitofrontal lesions impair use of cue-outcome associations in a devaluation task. Behav Neurosci 119:317-322.

Pompeiano M, Palacios JM, Mengod G (1994) Distribution of the serotonin 5-HT2 receptor family mRNAs: comparison between 5HT2A and 5-HT2C receptors. Brain Res Mol Brain Res 23:163178.

Robinson ES, Dalley JW, Theobald DE, Glennon JC, Pezze MA, Murphy ER, Robbins TW (2008) Opposing roles for 5-HT2A and 5-HT2C receptors in the nucleus accumbens on inhibitory response control in the 5 -choice serial reaction time task. Neuropsychopharmacology 33:2398-2406.

Robinson TE, Berridge KC (1993) The neural basis of drug craving: an incentive-sensitization theory of addiction. Brain Res Brain Res Rev 18:247-291.
Saunders BT, Robinson TE (2010) A cocaine cue acts as an incentive stimulus in some but not others: implications for addiction. Biol Psychiatry 67:730-736.

Saunders BT, Robinson TE (2013) Individual variation in resisting temptation: implications for addiction. Neurosci Biobehav Rev 37:1955-1975.

Schoenbaum G, Nugent SL, Saddoris MP, Setlow B (2002) Orbitofrontal lesions in rats impair reversal but not acquisition of go, no-go odor discriminations. Neuroreport 13:885-890.

Stevens L, Betanzos-Espinosa P, Crunelle CL, Vergara-Moragues E, Roeyers H, Lozano O, Dom G, Gonzalez-Saiz F, Vanderplasschen W, Verdejo-García A, Pérez-García M (2013) Disadvantageous decision-making as a predictor of drop-out among cocaine-dependent individuals in long-term residential treatment. Front Psychiatry 4:149.

Swinford-Jackson SE, Anastasio NC, Fox RG, Stutz SJ, Cunningham KA (2016) Incubation of cocaine cue reactivity associates with neuroadaptations in the cortical serotonin (5-HT) 5HT2C receptor (5-HT2CR) system. Neuroscience 324:50-61.

Winstanley CA, Tremblay M (2016) Risky choice on a cued gambling task in rats is not associated with elevated responding for conditioned reinforcement. San Diego: Society for Neuroscience.

Winstanley CA, Theobald DE, Dalley JW, Glennon JC, Robbins TW (2004) 5-HT2A and 5-HT2C receptor antagonists have opposing effects on a measure of impulsivity: interactions with global 5-HT depletion. Psychopharmacology (Berl) 176:376-385.

Worbe Y, Palminteri S, Savulich G, Daw ND, Fernandez-Egea E, Robbins TW, Voon V (2016) Valence-dependent influence of serotonin depletion on model-based choice strategy. Mol Psychiatry 21:624-629.

Zeeb FD, Winstanley CA (2011) Lesions of the basolateral amygdala and orbitofrontal cortex differentially affect acquisition and performance of a rodent gambling task. J Neurosci 31:2197-2204.

Zeeb FD, Winstanley CA (2013) Functional disconnection of the orbitofrontal cortex and basolateral amygdala impairs acquisition of a rat gambling task and disrupts animals' ability to alter decisionmaking behavior after reinforcer devaluation. J Neurosci 33:64346443.

Zeeb FD, Robbins TW, Winstanley CA (2009) Serotonergic and dopaminergic modulation of gambling behavior as assessed using a novel rat gambling task. Neuropsychopharmacology 34:23292343.

Zeeb FD, Floresco SB, Winstanley CA (2010) Contributions of the orbitofrontal cortex to impulsive choice: interactions with basal levels of impulsivity, dopamine signalling, and reward-related cues. Psychopharmacology (Berl) 211:87-98.

Zilberman N, Lavidor M, Yadid G, Rassovsky Y (2019) Qualitative review and quantitative effect size meta-analyses in brain regions identified by cue-reactivity addiction studies. Neuropsychology 33:319-334. 